Bio - grafía. Escritos sobre la Biología y su Enseñanza. ISSN 2027-1034

Edición Extraordinaria. p.p. 737 - 744

Memorias del IX Encuentro Nacional de Experiencias en Enseñanza de la Biología y la

Educación Ambiental. IV Congreso Nacional de Investigación en Enseñanza de la Biología.

\title{
LAS PRÁCTICAS DE CAMPO, RECURSO DIDÁCTICO PARALA ENSEÑANZA DE LA BIOLOGÍA: ESTUDIO DE CASO EN ASIGNATURAS DE BIOLOGÍA DE LA LICENCIATURA EN EDUCACIÓN BÁSICACON ÉNFASIS EN CIENCIAS NATURALES Y EDUCACIÓN AMBIENTAL DE LA UNIVERSIDAD DE ANTIOQUIA
}

\section{FIELD PRACTICES, DIDACTIC RESOURCES FOR TEACHING OF BIOLOGY: CASE STUDY IN BIOLOGY SUBJECTS OF UNDERGRADUATE PROGRAM IN BASIC EDUCATION WITH EMPHASIS IN NATURAL SCIENCES AND ENVIRONMENTAL EDUCATION OF THE UNIVERSTYY OF ANTIOQUIA}

\author{
Roger Andrés Muñoz Vásquez \\ Juan Diego Cardona Restrepo
}

\section{RESUMEN}

Esta investigación supone un acercamiento al análisis de uno de los recursos didácticos más valorados, en el proceso de formación inicial del profesorado de ciencias naturales (Morcillo et al. 1998); Se trata de las Prácticas de Campo. Se analiza esta actividad a la luz de las concepciones de estudiantes de la Licenciatura en Educación Básica con énfasis en Ciencias Naturales y Educación Ambiental de la Universidad de Antioquía, en la ciudad de Medellín. Se contrastan los datos aportados por varias investigaciones referentes al tema (Hodson;1994; Morzillo et al 1998; Rodrigo et al 1999; Legarralde et al 2009; Amórtegui et al, 2010), con las percepciones identificadas mediante una encuesta aplicada a un grupo aleatorio de estudiantes de diferentes niveles académicos, dentro del plan de estudio de la Licenciatura.

Se indaga sobre particularidades de orden logístico, temporal, metodológico y temático, que sirven para categorizar las prácticas de campo, según los modelos pedagógicos y el modelo de profesor que propone las actividades de campo. Se trata entonces, de un acercamiento preliminar a esta actividad tan valorada desde la teoría, como desde la realidad, según algunos resultados obtenidos en este estudio; además se consigue identificar la importancia asignada por los estudiantes al trabajo práctico para su futuro desempeño profesional, orientando procesos de aprendizaje de las ciencias naturales; sin embargo, también se identifican algunas particularidades que indican el desfase entre expectativa y realidad, es decir, entre lo que los futuros profesores de ciencias esperan en cuanto a su formación integral y en cuanto a lo que reciben después de participar en las salidas de campo.

Los resultados de este estudio indican la necesidad de darle continuidad a esta investigación, analizando aspectos administrativos y propios de la planeación docente, de tal manera que permita comprobar algunas de las hipótesis presentadas en el presente reporte. 
Bio - grafía. Escritos sobre la Biología y su Enseñanza. ISSN 2027-1034

Edición Extraordinaria. p.p. 737 - 744

Memorias del IX Encuentro Nacional de Experiencias en Enseñanza de la Biología y la

Educación Ambiental. IV Congreso Nacional de Investigación en Enseñanza de la Biología.

PALABRAS CLAVE: Prácticas de campo, Trabajo de campo, Formación de profesores de ciencias.

\begin{abstract}
This research is an approach to the analysis of one of the most valuable didactic resources in the process of initial training of sciences teachers (Morcillo et al., 1998). These are Field Practices. This activity is analyzed against the conceptions of students of the Basic Education Degree with emphasis in Sciences and Environmental Education of the University of Antioquia in the city of Medellin. The data provided by several investigations related to the subject are contrasted (Hodson;1994; Morzillo et al 1998; Rodrigo et al 1999; Legarralde et al 2009; Amórtegui et al, 2010) with the perceptions obtained through a survey applied to a random group of students of different academic levels within the curriculum of the Degree.
\end{abstract}

It's investigated on logistic, temporal, methodological and thematic particularities that serve to categorize the field practices according to the pedagogical models and the teacher's model that proposes the field activities. It's, of course, a preliminary approach to this activity as valued from theory, as from reality, according to some results obtained in this study, in addition it's possible to identify the importance assigned by students to the practical work for their future professional performance, orienting learning processes of Natural Sciences; However, there are also some particularities that indicate the gap between expectation and reality, that is, between what future sciences teachers expect in terms of their integral training and what they receive after participating in field trips.

The results of this study indicate the need to give continuity to this research by analyzing administrative and proper aspects of teaching planning in order to verify some of the hypotheses presented in this report.

KEY WORDS: Field Practices, Field work, Training of science teachers

\title{
INTRODUCCIÓN
}

La calidad del recurso humano en general y de los docentes en particular, está dada por la capacidad de respuesta eficiente a las situaciones cambiantes del medio, donde no se producen cambios en todos los ámbitos sino en las variables que conforman el sistema social o natural. Este fenómeno representa complejos problemas para la sociedad y los individuos. Uno de ellos es el de las competencias laborales, propias de los profesionales de la educación. En las condiciones del cambio tecnológico actual, la inversión en la formación y capacitación de los profesores se traduce en una mayor eficiencia y calidad en todos los procesos. El profesor debe estar en la capacidad de diseñar, ejecutar, evaluar y mejorar sus metodologías de manera constante acorde a las dinámicas culturales y tecnológicas contextuales y globales. Una de estas metodologías son las 
Bio - grafía. Escritos sobre la Biología y su Enseñanza. ISSN 2027-1034

Edición Extraordinaria. p.p. 737 - 744

Memorias del IX Encuentro Nacional de Experiencias en Enseñanza de la Biología y la

Educación Ambiental. IV Congreso Nacional de Investigación en Enseñanza de la Biología.

prácticas de campo, que exigen al docente una serie conocimientos en el ámbito procedimental, conceptual y actitudinal frente a un conjunto de conceptos específicos que aborda en sus clases (Morcillo et al. 1998).

Las prácticas de campo (en adelante $\mathrm{PC}$ ), utilizadas como recurso didáctico en el proceso de formación inicial de los profesores de biología, son muy valoradas en la medida que favorece el aprendizaje de conceptos, constituye una fuente de información directa, permite la ejemplificación y la experiencia contextualizada (Morcillo et al 1998). Es así que, para el docente en formación la realización de PC, constituye un factor importante en su futuro desempeño laboral, se debería esperar entonces la existencia de múltiples estudios de caracterización, análisis y sistematización en los centros de formación inicial del profesorado de ciencias, sin embargo, esto no es así. A nivel global la cantidad de estudios referentes a esta temática son bastante reducidos (Amórtegui et al 2010). En esta investigación se pretende realizar una introducción a la situación actual de las PC realizadas en el programa de la Licenciatura en Educación Básica con énfasis en Ciencias Naturales y Educación Ambiental de la Universidad de Antioquia de la ciudad de Medellín (Colombia), en el cual se realiza una caracterización de las prácticas de campo y la identificación de los roles del estudiante y del docente en estas actividades.

\section{METODOLOGÍA}

Esta investigación se enmarca en la perspectiva metodológica interpretativa, se realiza una aproximación a las PC, a partir de las percepciones del estudiantado participante en dichas propuestas didácticas. Dentro de la perspectiva metodológica de corte interpretativo se puede profundizar indicando que se trata de un estudio de caso, pues se estudia un fenómeno en su contexto y según Yin (1994), una investigación de este tipo se caracteriza por tener ciertas particularidades técnicas en las cuales hay muchas variables de interés, por este motivo se puede contar con múltiples fuentes de evidencias.

En esta investigación se toma la postura de Stake (1994), interpretada claramente por Crespo (2000, p.210): "el estudio de caso es una mezcla de retórica, diálogo, inducción, intuición y razonamiento". El diálogo e interpretación que se hace en este trabajo es sobre las concepciones y particularidades de las PC del núcleo de biología, de la Licenciatura en Educación Básica con Énfasis en Ciencias Naturales y Educación Ambiental de la Universidad de Antioquía.

Según la finalidad, se trata de una investigación aplicada en tanto que, pretende aportar datos relevantes para apoyar el diseño y ejecución de las prácticas de campo en el proceso de formación de profesorado en ciencias naturales de la Universidad de Antioquia.

El alcance temporal es de carácter sincrónico (Arnal et al, 1992), pues se trabajan con datos puntuales temporalmente, se indaga a los estudiantes participantes sobre sus percepciones y expectativas de las PC en su proceso de formación profesional, considerando las prácticas llevadas en su formación, no se trabaja con datos de 
Bio - grafía. Escritos sobre la Biología y su Enseñanza. ISSN 2027-1034

Edición Extraordinaria. p.p. 737 - 744

Memorias del IX Encuentro Nacional de Experiencias en Enseñanza de la Biología y la

Educación Ambiental. IV Congreso Nacional de Investigación en Enseñanza de la Biología.

históricos de la realización de PC en el programa académico.

Se utiliza como instrumento una encuesta con 14 preguntas dividida en tres dominios (identificación, tipos de PC y expectativas frente a la PC), esta se envía vía correo electrónico a todos los estudiantes de la licenciatura, en un formulario de Google, y se reciben respuestas a lo largo de unos dos meses, se seleccionan 30 encuestas considerando una distribución equitativa de estudiantes por nivel académico.

El instrumento permite identificar el nivel académico del estudiante, los cursos en los que se han realizado PC, la frecuencia de estas actividades, la confrontación de expectativas y realidad frente a las PC y los tipos de PC que se realizan en las asignaturas del núcleo de biología.

Con la información de las encuestas, se procede a interpretar los resultados, a la luz de la clasificación las PC y tipificar los roles del profesor y del estudiante en cada tipo de PC, realizada por Fernández y Elortegui, (1996) (citados por Morcillo et al, 1999, p. 267). Se llega entonces a la clasificación de las PC y a la identificación (preliminar) de los roles del estudiante y del profesor en estas actividades, disponiendo entonces de información para iniciar el análisis de la efectividad de este tipo de actividades, en la formación de licenciados en ciencias naturales.

\section{DISCUSIÓN Y CONCLUSIONES}

Según las apreciaciones de un grupo aleatorio de estudiantes de la Licenciatura en Educación Básica con énfasis en Ciencias Naturales y Educación Ambiental de la Universidad de Antioquia, se puede afirmar que las prácticas de campo, son una estrategia formativa valorada dentro del núcleo de biología, como un recurso que permite el fortalecimiento de saberes propios de la biología, específicamente en lo procedimental, más que en lo conceptual y en segundo nivel, se valora su potencialidad en aspectos propios del desempeño, como futuros profesores de ciencias naturales.

De igual manera, se puede clasificar al profesorado del mencionado núcleo de biología, específicamente en aquellas asignaturas donde se realizan PC, dentro de los modelos Transmisor, Tecnólogo e incluso el Artesano (Rodrigo et al,1999). Este modelo de profesor propone en las prácticas de campo, actividades direccionadas hacia las demostraciones y ejemplificaciones de conceptos o teorías; el estudiante toma por tanto un rol secundario de copista o toma de datos, que corroboran lo trabajado en las clases teóricas.

La encuesta aplicada a los estudiantes permite identificar cierto malestar frente a los tipos de prácticas de campo, desplegadas en las asignaturas del núcleo de biología. Esta situación, se da debido a la incongruencia del enfoque constructivista, crítico e investigativo, referente dominante en el programa académico, en relación con las actividades de tipo demostrativas, donde el estudiante toma un rol de copista en la mayoría de salidas de campo. Esta situación indica la necesidad de establecer líneas claras para el diseño y ejecución de prácticas de campo, a la luz de los pilares didácticos 
Bio - grafía. Escritos sobre la Biología y su Enseñanza. ISSN 2027-1034

\section{Edición Extraordinaria. p.p. 737 - 744}

Memorias del IX Encuentro Nacional de Experiencias en Enseñanza de la Biología y la Educación Ambiental. IV Congreso Nacional de Investigación en Enseñanza de la Biología.

del proceso de formación y las bases teóricas que se desean fortalecer en las prácticas profesionales de los futuros docentes de ciencias. Este hallazgo significa también, la necesidad de capacitación del profesorado actual de las asignaturas del núcleo de biología para planear, ejecutar y evaluar sus PC, considerando además de los objetivos específicos dentro de su campo de saber, los objetivos del programa de formación de licenciados (as) en ciencias naturales, además de un reconocimiento profundo de la importancia de las PC, fuera del fortalecimiento conceptual del saber específico, ampliando el campo visual hacia los saberes didácticos y procedimentales, que se pueden fortalecer con estas actividades en las que se pueden incorporar discusiones 0 contenidos que a su vez, lleven a los estudiantes a pensarse a sí mismos, como futuros diseñadores y ejecutores de este tipo de actividades en los diferentes contextos, que se pueden encontrar en la educación básica y media.

Se trata entonces de reconocer que las PC, van mucho más allá del saber específico y se deben tomar como una herramienta útil para fortalecer el dominio procedimental, pedagógico y didáctico del futuro profesorado de ciencias naturales.

Al categorizar las PC, haciendo énfasis en la identificación de un modelo didáctico y un modelo de profesor, surge la necesidad de trascender las apreciaciones de estudiantes y completar el análisis con el estudio de procedimientos administrativos, guías de campo, entrevistas a docentes y observación directa de esta propuesta metodológica. Se requiere la ampliación de esta investigación con instrumentos que impliquen el estudio de la importancia de las PC, asignado por el estamento administrativo. Dichos resultados podrían justificar algunos de los resultados de este trabajo; es el caso del rol del estudiante identificado, es decir, se podría sospechar que el rol de copista que se pudo identificar, se deba al escaso espacio que la administración del programa académico le asigna a las PC (evidenciable en el número de PC que los estudiantes manifiestan se realizan en las diferentes asignaturas), al igual que los tipos de docentes que corresponden a los de transmisores o tecnólogos puede deberse a que la mayoría de profesores se interesan exclusivamente por el saber específico, dejando de lado las potencialidades de las PC para abordar y fortalecer dominios conceptuales propios de la didáctica de las ciencias, entre otras posibilidades de no menos valor académico, como el análisis de procedimientos, análisis de contextos escolares y otros aspectos de gran valor, en el futuro desempeño profesional del estudiantado.

Se abren entonces diferentes líneas de investigación en este campo, todas ellas enmarcadas en el estudio del contexto de las PC, es decir, reconociendo múltiples aristas, tales como las dinámicas administrativas, la capacitación de docentes, el conocimiento del proceso general de formación por parte de los docentes y la utilización de este conocimiento en la planeación y ejecución de sus actividades. Lo que más apremia es la confirmación de la hipótesis planteada: los profesores de biología no reconocen la potencialidad de sus espacios conceptuales, para el fortalecimiento del saber didáctico y metodológico y consecuentemente no proponen actividades que apunten a este objetivo; a partir de los resultados de este trabajo se podría intuir que esto es así y que el profesorado del núcleo de biología trabaja sin reconocer adecuadamente su contexto: la formación disciplinar para la enseñanza de las ciencias. 
Bio - grafía. Escritos sobre la Biología y su Enseñanza. ISSN 2027-1034

Edición Extraordinaria. p.p. 737 - 744

Memorias del IX Encuentro Nacional de Experiencias en Enseñanza de la Biología y la

Educación Ambiental. IV Congreso Nacional de Investigación en Enseñanza de la Biología.

Con el propósito de ampliar o generar nuevas líneas de investigación, es imperativo, se hace necesario implementar instrumentos, que incluyan la participación masiva de estudiantes que aporten datos para una mayor descripción de las PC, de un programa de formación de profesorado en ciencias naturales. Se requiere el estudio particularizado de las fases de las PC, es decir, la planeación, ejecución y evaluación estas actividades, a la luz de los objetivos estipulados desde las asignaturas y del programa académico en general y también, desde las necesidades contextuales del futuro desempeño de los docentes de ciencias naturales.

El presente trabajo marca una ruta inicial. Se pretende hacer un especie de diagnóstico bajo el cual continuar, tanto la descripción de las PC en el proceso de formación del profesorado de ciencias, como la búsqueda de perspectivas en aras de identificar las características básicas que debe tener una actividad de este tipo, considerando las necesidades y realidades del profesorado en ciencias del contexto local y nacional. Consecuentemente se debe continuar en dos líneas de trabajo; una descriptiva en la cual se estudien las prácticas de campo a profundidad en sus dinámicas logísticas y múltiples dimensiones metodológicas, la categorización del profesorado del núcleo de biología, la importancia asignada desde la administración del programa y desde entes administrativos superiores, las expectativas del estudiantado y de sus futuros empleadores e incluso considerar el contexto donde en el futuro desarrollaran sus propias PC. La descripción del contexto de las PC se vislumbra tal vez como un cosmos en sí mismo, donde los múltiples factores podrían considerarse como objetos de investigación.

La otra línea sería la investigación experimental, que contribuya a la identificación de las especificaciones de la práctica necesaria en el proceso de formación de docentes de ciencias naturales. Este proceso requiere de insumos conceptuales procedentes del estudio del contexto y del análisis de los diferentes factores, que determinan el éxito o fracaso de estas actividades prácticas. Esta investigación implicaría además del estudio del contexto, la implementación de diferentes PC en grupos experimentales, el registro de datos de manera minuciosa, que puedan servir para establecer parámetros de éxito o fracaso y el estudio de percepciones de estudiantes y docentes, además de las posibles adaptaciones necesarias de procedimientos administrativos y logísticos. Se trata entonces de la búsqueda de nuevas formas o metodologías, que implicarían la mejora de las PC, realizadas en la formación del profesorado de ciencias naturales, donde se considere el saber científico (biológico), pedagógico y didáctico que se pueden optimizar con una herramienta metodológica tan rica en cuanto a sus múltiples potencialidades.

\section{REFERENCIAS BIBLIOGRÁFICAS}

Amórtegui, E., Correa, M. and Valbuena, E. (2010). Aporte de las prácticas de campo a la construcción de conocimiento profesional de futuros profesores de biología. In: II Congreso Internacional de Didáctica. [online] Girona: Universidad de Girona, pp.1-11. Available at: http://www.udg.edu/tabid/12826/language/es-ES/Default.aspx/ [Accessed 10 Feb. 2015]. 
Bio - grafía. Escritos sobre la Biología y su Enseñanza. ISSN 2027-1034

Edición Extraordinaria. p.p. 737 - 744

Memorias del IX Encuentro Nacional de Experiencias en Enseñanza de la Biología y la Educación Ambiental. IV Congreso Nacional de Investigación en Enseñanza de la Biología.

Anguita, F. y Ancochea, E. (1981). Prácticas de campo: alternativas a la excursión tradicional. I simposio Nacional sobre Enseñanza de la Geología (pp. 263-273). España: Vitoria-Gasteiz.

Arnal, J del Rincón D. y La Torre A. (1992). Investigación Educativa: Fundamentos y Metodología. Barcelona: Editorial Lapo.

Barberá, O. y Valdés, P. (1996). El trabajo práctico en la enseñanza de las ciencias: una revisión. Enseñanza de las Ciencias, 14 (3), 365-379.

Crespo, R. (2000). In: Second ISBEE World Congress "The Ethical Challenges of Globalization". São Paulo: Journal of Business Ethics, pp. 60-69 pp.http://link.springer.com/article/10.1023/A\%3A1017221129362.

De Pro, A. (1998). ¿Se pueden enseñar contenidos procedimentales en las clases de ciencias? Enseñanza de las Ciencias, 16 (1), 21-41.

Del Carmen, L. M. (1999). El estudio de Ecosistemas. Alambique, 20, 47-54.

Fernández, J. y Elortegui. N. (1996). Qué piensan los profesores acerca de cómo se debe enseñar. Enseñanza de las Ciencias, 14 (3), 331-342.

García, A. (2005). El jardín botánico como recurso didáctico. Revista Eureka sobre Enseñanza y Divulgación de las Ciencias, 2 (2), 209-217.

García, S., Martínez, M. y Móndelo, M. (1995). El trabajo práctico. Una intervención para la formación de profesores. Enseñanza de las Ciencias, 13 (2), 203-209.

García, S., Martínez, M. y Mondelo, M. (1998). Hacia la innovación de las actividades prácticas desde la formación del profesorado. Enseñanza de las Ciencias, 16 (2), 353366.

Hodson, D. (1994). Hacia un enfoque más crítico del trabajo de laboratorio. Enseñanza de las Ciencias, 12 (3), 299-313.

Hurtado, J. (2000). Metodología de la investigación holística. Venezuela: Sypal-Ivtc.

Izquierdo, M., Sanmartí, N. y Espinet, M. (1999). Fundamentación y diseño de las prácticas escolares de ciencias experimentales. Enseñanza de las Ciencias, 17 (1), 4559.

Legarralde, T., Vilches, A. and Darrigan, G. (2009). El trabajo de campo en la formación de los profesores de biología: una estrategia didáctica para mejorar la práctica docente. In: II Jornadas de Enseñanza e Investigación Educativa en el campo de las Ciencias Exactas y Naturales. [online] Buenos Aires: Universidad Nacional de la Plata, pp.165-170. Available at: http://jornadasceyn.fahce.unlp.edu. ar/iii-2012 [Accessed 10 Feb. 2015].

Morcillo, J., Rodrigo, M., Centeno, J. y Compani, M. (1998). Caracterización de las prácticas de campo: justificación y primeros resultados de una encuesta al profesorado. Enseñanza de las Ciencias de la Tierra, 63, 242-250. 
Bio - grafía. Escritos sobre la Biología y su Enseñanza. ISSN 2027-1034

Edición Extraordinaria. p.p. 737 - 744

Memorias del IX Encuentro Nacional de Experiencias en Enseñanza de la Biología y la Educación Ambiental. IV Congreso Nacional de Investigación en Enseñanza de la Biología.

Perales, F.J. (1994). Los trabajos prácticos y la didáctica de las ciencias. Enseñanza de las Ciencias, 12 (1), 122-125.

Pedrinaci, E., Sequerios, L., y García de la Torre, E. (1994). El trabajo de campo y el aprendizaje de la geología. Alambique: Didáctica de las Ciencias, 2, 37-45.

Pedrinaci, E. (2012). El trabajo de campo y la enseñanza de las ciencias. Alambique: Didáctica de las Ciencias Experimentales, 71, 81-89.

Rodrigo, M., Morcillo, J., Borges.R, Calvo, M., García, F. y Raviola, A. (1999). Concepciones sobre el trabajo práctico de campo (TPc): una aproximación al pensamiento de los futuros profesores. Revista Complutense de Educación, 10 (2), 261285.

Stake, R. E. (1994). Case study research: desing and methods. Thousand Oaks: Sage Publications. 